\title{
Nine-membered trans square planar chelates formed by a bisbi analogue
}

\author{
Michael R. Eberhard, ${ }^{a}$ Katie Heslop, ${ }^{b}$ A. Guy Orpen $^{\mathrm{b}}$ and Paul G. Pringle ${ }^{* b}$ \\ ${ }^{a}$ van 't Hoff Institute for Molecular Science, University of Amsterdam, Nieuwe \\ Achtergracht 166, 1018 WV Amsterdam, The Netherlands \\ ${ }^{b}$ School of Chemistry, University of Bristol, Cantocks Close, Bristol BS8 1TS, \\ United Kingdom \\ E-mail: paul.pringle@bristol.ac.uk
}

\section{General experimental details}

Unless otherwise stated, all reactions were carried out under a dry nitrogen atmosphere using standard Schlenk line techniques. Solvents were dried and deoxygenated by refluxing under a nitrogen atmosphere over appropriate drying agents: calcium hydride (for dichloromethane), sodium/benzophenone (THF). Phosphine 1 was stored under nitrogen at room temperature. Most complexes were stable to air in the solid state and were stored in air at room temperature. The Microanalytical Laboratory of the School of Chemistry, University of Bristol, carried out elemental analyses. The Mass Spectrometry Service, University of Bristol, recorded electron impact and Fast Atom Bombardment mass spectra on a MD800 and an Autospec. Infrared spectroscopy was carried out on a Perkin Elmer 1600 Series FTIR. ${ }^{31} \mathrm{P}\left\{{ }^{1} \mathrm{H}\right\},{ }^{13} \mathrm{C}\left\{{ }^{1} \mathrm{H}\right\}$ and ${ }^{1} \mathrm{H}$ NMR spectra were recorded on a Jeol $\Delta 300$ or Jeol GX400 at ambient temperature of the probe unless otherwise stated, using deuterated solvent to provide the field/frequency lock.

\section{Preparation of 1}

9-phosphabicyclo[3.3.1]nonane $(0.68 \mathrm{~g}, 4.80 \mathrm{mmol})$ was dissolved in THF $\left(9.6 \mathrm{~cm}^{3}\right)$, the solution was cooled to $-10{ }^{\circ} \mathrm{C}$ and $1.6 \mathrm{M} \mathrm{BuLi}\left(3 \mathrm{~cm}^{3}, 4.80 \mathrm{mmol}\right)$ was added dropwise. After stirring for $15 \mathrm{~min}$, a solution of 2,2'-biphenyldimethanol di-p-tosylate (1.26 g, $2.40 \mathrm{mmol})$ in THF $\left(4.8 \mathrm{~cm}^{3}\right)$ was added dropwise. The reaction mixture was stirred for 
$10 \mathrm{~h}$. The solvent was removed in vacuum and toluene $\left(30 \mathrm{~cm}^{3}\right)$ and deoxygenated water $\left(20 \mathrm{~cm}^{3}\right)$ was added to the solid residue. After stirring the biphasic system vigorously, the organic phase was separated and the aqueous phase was extracted three times with toluene $\left(15 \mathrm{~cm}^{3}\right.$ each). The combined organic phases were dried over $\mathrm{MgSO}_{4}$ and filtered over basic alumina. Removal of the solvent in vacuum gave pure $\mathbf{1}$ $(1.00 \mathrm{~g}, 2.16 \mathrm{mmol}, 90 \%)$ as a colorless solid.

$$
\begin{array}{ll}
\delta_{\mathrm{P}}\left(162 \mathrm{MHz} ; \mathrm{C}_{6} \mathrm{D}_{6}\right) & -27.0 \\
\delta_{\mathrm{H}}\left(400 \mathrm{MHz} ; \mathrm{CD}_{2} \mathrm{Cl}_{2}\right) & 7.37-7.22(4 \mathrm{H}, \mathrm{m}, \mathrm{ArH}), 7.22-7.14(4 \\
& \mathrm{H}, \mathrm{m}, \mathrm{ArH}), 2.84\left(2 \mathrm{H},{ }^{2} J(\mathrm{HH}) 13.9 \mathrm{~Hz},\right. \\
& \left.{ }^{2} J(\mathrm{PH}) 2.0 \mathrm{~Hz}, \mathrm{ABX}, \mathrm{PCH}_{2}\right), 2.71(2 \mathrm{H}, \\
& { }^{2} J(\mathrm{HH}) 13.9 \mathrm{~Hz},{ }^{2} J(\mathrm{PH}) 2.0 \mathrm{~Hz}, \mathrm{ABX}, \\
& \left.\mathrm{PCH}_{2}\right), 2.12-1.83(14 \mathrm{H}, \mathrm{m}), 1.79-1.40 \\
& (14 \mathrm{H}, \mathrm{m}) \\
& 141-125(\operatorname{aromatic} \text { carbons }), 32.3(\mathrm{~d}, \\
\delta_{\mathrm{C}}\left(100 \mathrm{MHz} ; \mathrm{CD}_{2} \mathrm{Cl}_{2}\right) & J(\mathrm{PC}) 9.8 \mathrm{~Hz}), 32.2(\mathrm{~d}, J(\mathrm{PC}) 9.8 \mathrm{~Hz}), \\
& 28.0(\mathrm{~d}, J(\mathrm{PC}) 1.5 \mathrm{~Hz}), 27.7(\mathrm{~d}, J(\mathrm{PC}) 1.5 \\
& \mathrm{Hz}), 25.0(\mathrm{~d}, J(\mathrm{PC}) 7.4 \mathrm{~Hz}), 24.9(\mathrm{~s}), 24.7 \\
& (\mathrm{~d}, J(\mathrm{PC}) 8.9 \mathrm{~Hz}), 24.6(\mathrm{~s}), 23.4(\mathrm{~d}, J(\mathrm{PC})
\end{array}
$$$$
5.4 \mathrm{~Hz}), 21.7(\mathrm{~s})
$$

\section{Preparation of 2}

$1(0.141 \mathrm{~g}, 0.305 \mathrm{mmol})$ was dissolved in dichloromethane $\left(6 \mathrm{~cm}^{3}\right)$ and added slowly dropwise to a solution of $\left[\mathrm{RhCl}(\mathrm{CO})_{2}\right]_{2}(0.056 \mathrm{~g}, 0.145 \mathrm{mmol})$ in dichloromethane $(6$

$\mathrm{cm}^{3}$ ). The solution was stirred for $30 \mathrm{~min}$ after which the solvent was removed in vacuum to give $2(0.182 \mathrm{~g}, 0.290 \mathrm{mmol}, 100 \%)$ as a yellow solid.

$$
\begin{array}{ll}
\delta_{\mathrm{P}}\left(121 \mathrm{MHz} ; \mathrm{CD}_{2} \mathrm{Cl}_{2}\right) & 22.1\left({ }^{1} J\left(\mathrm{RhP}_{\mathrm{A}}\right) 122 \mathrm{~Hz},{ }^{2} J\left(\mathrm{P}_{\mathrm{A}} \mathrm{P}_{\mathrm{B}}\right) 350 \mathrm{~Hz}\right), \\
& 15.4\left({ }^{1} J\left(\mathrm{RhP}_{\mathrm{B}}\right) 122 \mathrm{~Hz},{ }^{2} J\left(\mathrm{P}_{\mathrm{A}} \mathrm{P}_{\mathrm{B}}\right) 350 \mathrm{~Hz}\right) \\
& 7.32-7.11(8 \mathrm{H}, \mathrm{m}), 3.83-3.38(3 \mathrm{H}, \mathrm{m}), \\
\delta_{\mathrm{H}}\left(300 \mathrm{MHz} ; \mathrm{CD}_{2} \mathrm{Cl}_{2}\right) & 2.81-1.61(29 \mathrm{H}, \mathrm{m})
\end{array}
$$


$\delta_{\mathrm{C}}\left(75 \mathrm{MHz} ; \mathrm{CD}_{2} \mathrm{Cl}_{2}\right)$

\begin{tabular}{|c|c|}
\hline & $\left.\mathrm{Hz},{ }^{2} J(\mathrm{PC}) 15.6 \mathrm{~Hz}\right), 141.7(\mathrm{~d}, J(\mathrm{PC}) 4.5$ \\
\hline & $\mathrm{Hz}), 141.6(\mathrm{~d}, J(\mathrm{PC}) 4.2 \mathrm{~Hz}), 140.9(\mathrm{dd}$ \\
\hline & $J(\mathrm{PC}) 4.4 \mathrm{~Hz}, J(\mathrm{PC}) 1.0 \mathrm{~Hz}), 136.1(\mathrm{q})$ \\
\hline & $134.5(\mathrm{t}, J(\mathrm{PC}) 1.3 \mathrm{~Hz}), 131.7(\mathrm{~d}, J(\mathrm{PC})$ \\
\hline & $3.8 \mathrm{~Hz}), 131.5(\mathrm{~d}, J(\mathrm{PC}) 2.8 \mathrm{~Hz}), 131.3$ \\
\hline & $(\mathrm{d}, J(\mathrm{PC}) 2.4 \mathrm{~Hz}), 130.5(\mathrm{~d}, J(\mathrm{PC}) 4.1$ \\
\hline & $\mathrm{Hz}), 127.0(\mathrm{~d}, J(\mathrm{PC}) 2.4 \mathrm{~Hz}), 125.78(\mathrm{~d}$ \\
\hline & $J(\mathrm{PC}) 4.2 \mathrm{~Hz}), 125.78(\mathrm{~d}, J(\mathrm{PC}) 1.7 \mathrm{~Hz})$ \\
\hline & $31.2(\mathrm{~d}, J(\mathrm{PC}) 3.8 \mathrm{~Hz}), 30.9(\mathrm{~d}, J(\mathrm{PC}) 4.5$ \\
\hline & $\mathrm{Hz}), 30.4(\mathrm{~d}, J(\mathrm{PC}) 4.8 \mathrm{~Hz}), 30.2(\mathrm{~d}$, \\
\hline & $J(\mathrm{PC}) 3.8 \mathrm{~Hz}), 29.7(\mathrm{~s}), 29.5(\mathrm{~d}, J(\mathrm{PC})$ \\
\hline & $1.0 \mathrm{~Hz}), 29.3(\mathrm{~d}, J(\mathrm{PC}) 1.4 \mathrm{~Hz}), 28.6(\mathrm{t}$ \\
\hline & $J(\mathrm{PC}) 2.1 \mathrm{~Hz}), 28.4(\mathrm{t}, J(\mathrm{PC}) 2.1 \mathrm{~Hz})$ \\
\hline & $28.1(\mathrm{~d}, J(\mathrm{PC}) 14.2 \mathrm{~Hz}), 26.9-26.7(\mathrm{~m})$ \\
\hline & $26.5(\mathrm{~m}), 24.5(\mathrm{t}, J(\mathrm{PC}) 2.1 \mathrm{~Hz}), 24.2(\mathrm{t}$ \\
\hline & $J(\mathrm{PC}) 2.4 \mathrm{~Hz}), 22.7(\mathrm{~d}, J(\mathrm{PC}) 2.4 \mathrm{~Hz})$ \\
\hline & $22.6(\mathrm{~d}, J(\mathrm{PC}) 2.1 \mathrm{~Hz}), 22.5(\mathrm{~d}, J(\mathrm{PC}) 3.1$ \\
\hline & $\mathrm{Hz}), 22.2(\mathrm{~d}, J(\mathrm{PC}) 2.8 \mathrm{~Hz}), 21.9(\mathrm{~d}$, \\
\hline & $J(\mathrm{PC}) 3.1 \mathrm{~Hz}), 21.8(\mathrm{~d}, J(\mathrm{PC}) 3.5 \mathrm{~Hz})$ \\
\hline & $19.7(\mathrm{~d}, J(\mathrm{PC}) 4.2 \mathrm{~Hz}), 19.5(\mathrm{~d}, J(\mathrm{PC}) 4.2$ \\
\hline & $\mathrm{Hz})$ \\
\hline$v(\mathrm{CO})(\mathrm{DCM})$ & $1957 \mathrm{~cm}^{-1}$ \\
\hline FAB mass spectrum: $\mathrm{m} / \mathrm{z}$ & $600\left(\mathrm{M}^{+}-\mathrm{H}-\mathrm{CO}\right)$ \\
\hline Elemental analysis & 7) $\mathrm{H} \cdot 608$ \\
\hline$[\mathrm{RhCl}(\mathrm{CO}) \mathbf{1}] \cdot 0.25 \mathrm{CH}_{2} \mathrm{Cl}_{2}$ &. \\
\hline
\end{tabular}

186.5 (ddd, ${ }^{1} J(\mathrm{RhC}) 74.9 \mathrm{~Hz},{ }^{2} J(\mathrm{PC}) 16.7$ , $141.6(\mathrm{~d}, J(\mathrm{PC}) 4.2 \mathrm{~Hz}), 140.9(\mathrm{dd}$, 134.5 (t, J(PC) $1.3 \mathrm{~Hz}), 131.7$ (d, J(PC)

3.8 Hz), $131.5(\mathrm{~d}, J(\mathrm{PC}) 2.8 \mathrm{~Hz}), 131.3$ $\mathrm{Hz}), 127.0$ (d, J(PC) $2.4 \mathrm{~Hz}), 125.78$ (d, (PC) $4.2 \mathrm{~Hz}), 125.78(\mathrm{~d}, J(\mathrm{PC}) 1.7 \mathrm{~Hz})$, $\mathrm{Hz}), 30.4$ (d, J(PC) $4.8 \mathrm{~Hz}), 30.2$ (d, (PC) $3.8 \mathrm{~Hz}), 29.7(\mathrm{~s}), 29.5(\mathrm{~d}, J(\mathrm{PC})$

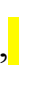
1.0 Hz), $29.3(\mathrm{~d}, J(\mathrm{PC}) 1.4 \mathrm{~Hz}), 28.6(\mathrm{t}$, 
pentane $\left(80 \mathrm{~cm}^{3}\right)$ was added. The yellow precipitate was filtered off, washed with pentane and dried in vacuum to give $3(0.048 \mathrm{~g}, 0.075 \mathrm{mmol}, 50 \%)$.

3 crystallised out as a solvate with one molecule of pentane. It was therefore only moderately soluble and no ${ }^{13} \mathrm{C}\left\{{ }^{1} \mathrm{H}\right\}$ NMR spectrum could be recorded. However, when a small sample of this complex was prepared and precipitated out with diethyl ether, no solvate is formed and the complex is readily soluble. Due to the small amount of sample prepared, no ${ }^{13} \mathrm{C}\left\{{ }^{1} \mathrm{H}\right\}$ NMR spectrum could be recorded either. The elemental analysis refers to this sample.

$\begin{array}{ll}\delta_{\mathrm{P}}\left(121 \mathrm{MHz} ; \mathrm{CD}_{2} \mathrm{Cl}_{2}\right) & 13.6(\mathrm{br} \mathrm{m}) \\ \delta_{\mathrm{H}}\left(300 \mathrm{MHz} ; \mathrm{CD}_{2} \mathrm{Cl}_{2}\right) & 7.49-7.05(8 \mathrm{H}, \mathrm{m}), 3.09(4 \mathrm{H}, \mathrm{br} \mathrm{s})(28 \\ & \mathrm{H}, \mathrm{m}) \\ \text { FAB mass spectrum: } \mathrm{m} / \mathrm{z} & 567\left(\mathrm{M}^{+}-2 \mathrm{H}-2 \mathrm{Cl}\right) \\ \text { Elemental analysis } & \mathrm{C}: 56.63(56.31) \mathrm{H}: 6.09(6.30)\end{array}$

\section{Preparation of trans-4}

$1(0.085 \mathrm{~g}, 0.184 \mathrm{mmol})$ was dissolved in acetone $\left(30 \mathrm{~cm}^{3}\right)$ and added dropwise to a solution of Zeise's salt $(0.066 \mathrm{~g}, 0.179 \mathrm{mmol})$ in acetone $\left(30 \mathrm{~cm}^{3}\right)$. A colorless precipitate formed and the solution was stirred for 15 min after which the solvent was removed. The suspension was filtered over celite to remove precipitated potassium chloride. The celite was washed with dichloromethane and the solvent was removed in vacuum to give $\mathbf{4}$ $(0.130 \mathrm{~g}, 0.178 \mathrm{mmol}, 100 \%)$ as a yellow solid.

The complex appeared to form a solvate-complex in concentrated $\mathrm{CD}_{2} \mathrm{Cl}_{2}$ solutions, which precipitated out. A satisfactory ${ }^{13} \mathrm{C}\left\{{ }^{1} \mathrm{H}\right\}$ NMR measurement was therefore not possible.
$\delta_{\mathrm{P}}\left(121 \mathrm{MHz} ; \mathrm{CD}_{2} \mathrm{Cl}_{2}\right)$
$9.9\left(\mathrm{~s},{ }^{1} J(\mathrm{PtP}) 2500 \mathrm{~Hz}\right)$
$\delta_{\mathrm{H}}\left(300 \mathrm{MHz} ; \mathrm{CD}_{2} \mathrm{Cl}_{2}\right)$
7.36 - $7.12(8 \mathrm{H}, \mathrm{m}), 3.83$ - $3.38(2 \mathrm{H}, \mathrm{m})$,
2.88 - 1.07 (30 H, m)
FAB mass spectrum: $\mathrm{m} / \mathrm{z}$ $728\left(\mathrm{M}^{+}\right), 691\left(\mathrm{M}^{+}-2 \mathrm{H}-\mathrm{Cl}\right), 655\left(\mathrm{M}^{+}-\right.$ $2 \mathrm{H}-2 \mathrm{Cl}$ ) 


\section{Preparation of cis-5}

$1(0.112 \mathrm{~g}, 0.242 \mathrm{mmol})$ was dissolved in dichloromethane $\left(5 \mathrm{~cm}^{3}\right)$ and added dropwise to a solution of $\left[\mathrm{PtCl}_{2}(\mathrm{cod})\right](0.091 \mathrm{~g}, 0.242 \mathrm{mmol})$ in dichloromethane $\left(5 \mathrm{~cm}^{3}\right)$. The solution was stirred for $1 \mathrm{~h}$ after which the solvent was removed to a minimum and diethyl ether $\left(80 \mathrm{~cm}^{3}\right)$ was added. The colorless precipitate, which formed, was filtered off, washed with diethyl ether and dried in vacuum to give $5(0.059 \mathrm{~g}, 0.150$ mmol, 85\%).

$\delta_{\mathrm{P}}\left(162 \mathrm{MHz} ; \mathrm{CD}_{2} \mathrm{Cl}_{2}\right)$

$\delta_{\mathrm{H}}\left(300 \mathrm{MHz} ; \mathrm{CD}_{2} \mathrm{Cl}_{2}\right)$

$\delta_{\mathrm{C}}\left(75 \mathrm{MHz} ; \mathrm{CD}_{2} \mathrm{Cl}_{2}\right)$ $1.6\left(\mathrm{~d},{ }^{1} J\left(\mathrm{PtP}_{\mathrm{A}}\right) 3549 \mathrm{~Hz},{ }^{2} J\left(\mathrm{P}_{\mathrm{A}} \mathrm{P}_{\mathrm{B}}\right) 16\right.$

$\mathrm{Hz}),-3.5\left(\mathrm{~d},{ }^{1} J\left(\mathrm{PtP}_{\mathrm{B}}\right) 3746 \mathrm{~Hz},{ }^{2} J\left(\mathrm{P}_{\mathrm{A}} \mathrm{P}_{\mathrm{B}}\right)\right.$ $16 \mathrm{~Hz}$ ) 7.58 - $7.07(8 \mathrm{H}, \mathrm{m}), 4.05-1.07(32 \mathrm{H}$, $\mathrm{m})$

142.4 (dd, J(PC) 4.2 Hz, J(PC) $1.0 \mathrm{~Hz}$ ), 139.9 (dd, J(PC) $4.5 \mathrm{~Hz}, J(\mathrm{PC}) 1.0 \mathrm{~Hz})$, 136.5 (d, J(PC) 2.8 Hz), 135.0 (d, J(PC) $6.6 \mathrm{~Hz}), 131.7$ (d, J(PC) $5.5 \mathrm{~Hz}), 131.6$ (dd, J(PC) $2.8 \mathrm{~Hz}, J(\mathrm{PC}) 1.0 \mathrm{~Hz}), 130.8$ (d, J(PC) $4.1 \mathrm{~Hz}), 128.7$ (d, J(PC) 3.5 $\mathrm{Hz}), 128.3$ (d, J(PC) $2.8 \mathrm{~Hz}), 127.73$ (d, $J(\mathrm{PC}) 3.1 \mathrm{~Hz}), 127.66(\mathrm{~d}, J(\mathrm{PC}) 1.7 \mathrm{~Hz})$, 126.8 (d, J(PC) 3.1 Hz), 33.6 (s), 33.2 (s), $32.0(\mathrm{~d}, J(\mathrm{PC}) 1.4 \mathrm{~Hz}), 28.7$ (dd, J(PC) $22.5 \mathrm{~Hz}, J(\mathrm{PC}) 1.4 \mathrm{~Hz}), 28.4$ (s), 28.0 (d, $J(\mathrm{PC}) 5.5 \mathrm{~Hz}), 27.5(\mathrm{~d}, J(\mathrm{PC}) 4.8 \mathrm{~Hz})$, 27.3 (s), 27.2 (s), 26.91 (s), 26.86 (s), $26.6(\mathrm{~d}, J(\mathrm{PC}) 5.2 \mathrm{~Hz}), 24.1(\mathrm{dd}, J(\mathrm{PC})$ $28.0 \mathrm{~Hz}, J(\mathrm{PC}) 1.0 \mathrm{~Hz}), 21.9$ (d, J(PC) 6.9 Hz), 21.6 (d, J(PC) 5.2 Hz), 20.3 (dd, 
$J(\mathrm{PC}) 11.8 \mathrm{~Hz}, J(\mathrm{PC}) 4.5 \mathrm{~Hz})$

$\delta_{\mathrm{P}}\left(86 \mathrm{MHz} ; \mathrm{CD}_{2} \mathrm{Cl}_{2}\right)$

$154.5\left(\mathrm{dd},{ }^{1} J\left(\mathrm{PtP}_{\mathrm{A}}\right) 3746 \mathrm{~Hz},{ }^{1} J\left(\mathrm{PtP}_{\mathrm{B}}\right)\right.$

$3549 \mathrm{~Hz})$

FAB mass spectrum: $\mathrm{m} / \mathrm{z}$

$728\left(\mathrm{M}^{+}\right), 693\left(\mathrm{M}^{+}-\mathrm{Cl}\right), 655\left(\mathrm{M}^{+}-2 \mathrm{H}-\right.$ $2 \mathrm{Cl})$

Elemental analysis

C: 50.55 (49.46) H: 6.04 (5.53) 